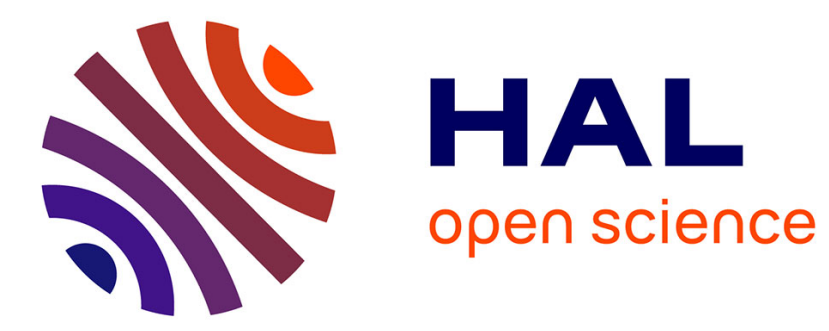

\title{
Neutron reflectivity and surface tension measurements of a diblock copolymer monolayer on a selective solvent
}

\author{
M. Kent, L. Lee, B. Factor, F. Rondelez, G. Smith
}

\section{To cite this version:}

M. Kent, L. Lee, B. Factor, F. Rondelez, G. Smith. Neutron reflectivity and surface tension measurements of a diblock copolymer monolayer on a selective solvent. Journal de Physique IV Proceedings, 1993, 03 (C8), pp.C8-49-C8-52. 10.1051/jp4:1993810 . jpa-00252229

\section{HAL Id: jpa-00252229 \\ https://hal.science/jpa-00252229}

Submitted on 1 Jan 1993

HAL is a multi-disciplinary open access archive for the deposit and dissemination of scientific research documents, whether they are published or not. The documents may come from teaching and research institutions in France or abroad, or from public or private research centers.
L'archive ouverte pluridisciplinaire HAL, est destinée au dépôt et à la diffusion de documents scientifiques de niveau recherche, publiés ou non, émanant des établissements d'enseignement et de recherche français ou étrangers, des laboratoires publics ou privés. 


\title{
Neutron reflectivity and surface tension measurements of a diblock copolymer monolayer on a selective solvent
}

\author{
M.S. KENT, L.T. LEE* , B.J. FACTOR ${ }^{* *}$, F. RONDELEZ ${ }^{* *}$ and G. SMITH*** \\ Dept. 1815, Sandia National Laboratories, P.O. Box 5800, Albuquerque, NM, U.S.A. \\ ${ }^{*}$ Laboratoire Léon Brillouin, CE-Saclay, 91191 Gif sur Yvette, France \\ ** Laboratoire P.S.I., URA 1379 du CNRS, Institut Curie, Section de Physique et Chimie, 11 nue Pierre et \\ Marie Curie, 75005 Paris, France \\ ${ }_{* * *}^{*}$ LANSCE, Los Alamos National Laboratories, Los Alamos, NM, U.S.A.
}

\begin{abstract}
We report measurements of the concentration profile of the submerged block of a diblock copolymer monolayer at the air surface of a selective solvent. The profile is found to contain a depletion layer near the surface $(\sim 35 \AA)$, followed by a parabolic (or similar) decay which tails off smoothly away from the surface. The dependencies of the maximum dimension $\left(\mathrm{h}^{*}\right)$ of the profile on the surface density $(\sigma)$ and the molecular weight (M) of the submerged block are found to be weaker than those predicted in the limit of strong stretching, but are in reasonably good agreement with numerical SCF calculations of Whitmore, et al. A sharp rise in the surface pressure (measured simultaneous with the reflectivity) is observed for each sample at high surface densities which is not predicted by the existing mean-field or scaling theories.
\end{abstract}

\section{Introduction}

Diblock copolymers tend to locate at interfaces and thus have great potential for use as surface or interfacial modifiers. The liquid-air interface is a model interface which allows the key variable, the surface coverage, to be varied continuously. In addition, the essential physical characteristics of the layer, the segmental concentration profile and the free energy of interaction of the submerged blocks, can be probed simultaneously in a straightforward manner by neutron reflectivity and surface tension measurements $(1,2)$. Information about block copolymers at this model interface will be applicable to important industrial problems such as the use of block copolymers to enhance adhesion between a polymeric matrix and a solid surface, to control the domain size in incompatible polymer blends, and to stabilize colloidal dispersions.

\section{Experimental}

In this study we have used polydimethylsiloxane-polystyrene (PDMS-PS) diblock copolymers which form a monolayer at the air surface of ethyl benzoate (EB), a selective, good solvent for PS. Two highly asymmetric PDMS-PS diblock copolymers with a perdeuterated PS block were studied: 4.5-60 (Polymer Labs, England) and 15-175 (Polymer Standard Service, Germany), where the numbers denote the molecular weights (in $\mathrm{kg} / \mathrm{mole}$ ) of the PDMS and PS blocks, respectively. The polydispersity of each sample was 1.1 and both were used as received. Ethyl benzoate was purchased from Aldrich and vacuum distilled before use. The monolayers were formed by depositing a small dry grain of the copolymer onto the surface of the solvent. This avoided the use of an organic spreading solvent and the possibility of its partial retention in the monolayer. The surface density $\sigma$ was either increased by adding grains of polymer or decreased by aspirating the surface. Reflectivity measurements have been performed on the DESIR (LLB) and the SPEAR (Los Alamos Nat. Lab.) reflectometers. The accessible range of $q$ $(=4 \pi \sin \theta / \lambda)$ on DESIR for this system extends up to $0.04 \AA^{-1}$, while on SPEAR a larger range up to $0.10 \AA^{-1}$ is accessible. In both cases the critical edge for total reflection was obtained and used to normalize the data. The reflectivity data were collected for 4-6 hour periods for a total time of up to 16 hours. The stability of the layer was monitored by measuring the surface tension $(\gamma)$ before and after each reflectivity measurement. The surface pressure $\left(\gamma_{\mathrm{EB}}-\gamma\right)$ ranged between zero and $8 \mathrm{dynes} / \mathrm{cm}$ for the smallest and largest $\sigma$ values, respectively. 


\section{Detailed segmental concentration profile of the submerged PS block}

The large range of $q$ accessible on SPEAR allows very detailed information about the profile to be obtained, in this case the presence of a depletion layer near the surface and a smooth tail off away from the surface. A representative reflectivity curve from SPEAR for the 4.5-60 sample is shown in Figure 1a (surface pressure of 2.7 dyne/cm). At this surface pressure, the submerged blocks are well into the interacting regime. The data are plotted as the reflectivity from the monolayer covered surface divided by that for the pure solvent surface. The reflectivity for the pure solvent agrees well with the Fresnel curve with 0 to $3 \AA$ roughness. The solid curves are the best fits (minimization of $\chi^{2}$ ) obtained for the PS segmental concentration profiles shown in Figure 1b: a parabolic profile with an exponential tail (1), a parabolic profile with a depletion layer (2), and a parabolic profile with both an exponential tail and a depletion layer (3). The maximum in the concentration profile is roughly $11 \%$. In Figure $1 \mathrm{a}$, the experimental data fall below curve (1) for $q>0.04 \AA^{-1}$ indicating that a depletion layer (roughly $35 \AA$ ) is required to fit the data. The smooth tail is also required, as shown by the fact that curve (2) oscillates more than the experimental data, particularly around $q=0.02$ to $0.04 \AA^{-1}$. Thus these data show conclusively that the concentration profile of the dangling PS blocks contains both a depletion layer near the surface and a smooth tail-off away from the surface.

\section{Variation of $h^{*}$ with surface density $(\sigma)$ and PS block molecular weight (M)}

While the $q$ range accessible on DESIR is smaller than that on SPEAR, it is entirely adequate to obtain the gross characteristics of the profile. In Figure 2 , we have plotted $h^{*}$ (obtained from fits of a pure parabola to reflectivity data from DESIR), normalized by the radii of gyration $82 \AA$ and $154 \AA$, respectively, as a function of reduced surface density $\sigma^{*}\left(=\sigma \pi \mathrm{R}_{\mathrm{g}}{ }^{2}\right)$ for the 4.5-60 and 15-175 samples. Over the range $2<\sigma^{*}<11$, where the PS blocks are interacting, $h^{*}$ increases roughly $40 \%$. The increase appears to be roughly linear with $\sigma^{*}$ and may be described by: $\mathrm{h}^{*} / \mathrm{R}_{\mathrm{g}}=3.08\left(1+0.055 \sigma^{*}\right)$. This suggests that the amount of chain stretching increases roughly linearly with the degree of crowding among the PS blocks. In addition to the linear relation between $h * / R_{\mathrm{g}}$ and $\sigma^{*}$, the data can also be represented by the power law $h^{*} \sim$ $M^{0.86} \sigma^{0.22}$. We note that the dependencies on both variables in the experimental data are weaker than the values predicted in the limit of strong stretching where $h^{*} \sim M \sigma^{0.33}(3,4)$. Thus, we conclude that the limiting behavior is not obtained for $\sigma^{*}<11$. Numerical SCF calculations corresponding to the 4.5-60 and 15-175 copolymers have been performed by Whitmore, et al. (5) There are no adjustable parameters in the calculations (a Kuhn length of $7 \AA$ for PS in a good solvent was used), and the results, which are consistent with $\mathrm{h}^{*} \sim \mathrm{M}^{0.80} \sigma^{0.22}$, are shown as the curves in Figure 2. There is good agreement for the magnitude of $h^{*}$ and the dependence on $\sigma$. On the other hand, the dependence on $M$ in the calculations is slightly weaker than in the experimental data. This difference may be due to the neglect of the depletion layer in the fits of the DESIR data. While the fitted value of $h^{*}$ for the 4.5-60 data of Figure 1 is only $2 \%$ larger when a depletion layer is included than when it is neglected, the difference may vary with molecular weight, such that a small systematic error in the molecular weight dependence may result when the depletion layer is neglected.

\section{Surface pressure}

The surface pressure isotherms for the 4.5-60 and 15-175 copolymers are shown in Figure 3. The PDMS contribution to the surface pressure is very small over the accessible range of $\sigma(1)$, so the rapid rise in surface pressure in each case must be due to the interaction of the submerged PS blocks. This rapid rise in surface pressure is much steeper than is predicted by current meanfield or scaling theories for interacting tethered chains $(1,3,4)$. In fact, the part of the isotherm which rises steeply can be described by a Van der Waals gas model with hard core interactions:

$$
\Pi=\mathrm{kT} /\left(\sigma^{-1}-\mathrm{A}_{\mathrm{O}}\right)
$$

where $A_{0}$ is the hard core cross-sectional area. This is shown by the solid curves in Figure 3 . The values for the hard core radii are $47 \AA$ and $29 \AA$, for the 15-175 and 4.5-60 copolymers, respectively. These values may be compared to the corresponding dilute solution radii of gyration for the PS blocks, which are $154 \AA$ and $82 \AA$, respectively. We find that the distance between anchoring points when the hard core-like behavior occurs $\left(\vee \mathrm{A}_{\mathrm{o}}\right)$ is $54 \%$ and $62 \%$ of the dilute solution radii of gyration for the respective samples. We note that the layer with the higher molecular weight PS block is compressible to a somewhat greater degree. 


\section{Discussion}

Several studies of the segmental profiles of tethered chains have been reported recently (68). However, the simultaneous measurement of both the segmental concentration profile and the surface pressure, which is closely related to the free energy (1), provides a unique opportunity to test in great detail theories of chain interactions in tethered layers. The above results show that the variation of the maximum dimension of the profile with surface density and molecular weight is reasonably well described by the numerical SCF calculations of Whitmore, et al. However, the rapid rise in surface pressure which occurs at the very end of the accessible range of surface density is not predicted by this theory. In order to reconcile these two observations, we suggest the following model. The outer edges of the coils, where the segmental concentration is low, interpenetrate to some extent such that the interaction is well described by the SCF picture. However, as the chains are pushed closer together, interpenetration becomes impeded due to the uncrossibility of the chains. The chains are trapped in this state since it is difficult for a free end to retrace its path along the contour of the chain and escape from the constraints imposed by neighboring chains (9). Further compression leads to a squeezing of the coils and the rapid rise in surface pressure. Evidence for such a steric effect and an accompanying rapid rise in surface pressure has recently been suggested in molecular dynamics simulations (10) which explicitly take into account higher order interactions and the uncrossiblity of chains, and also in a recent theoretical treatment (11) which includes a finite volume for the chain segments.

[1] Kent, M.S., Lee, L.T., Farnoux, B., Rondelez, F. Macromolecules 25, (1992), 6240.

[2] Factor, B.J., Lee, L.T., Kent, M., Rondelez, F. submitted to Physical Review E

[3] Alexander S., J. Phys. France 38 (1977), 983.

[4] Milner, S., Witten, T., Cates, M.E. Macromolecules 21 (1988) 2610.

[5] Whitmore, M.D., Noolandi, J. Macromolecules 23 (1990), 3321. Whitmore, M.D.,

Baranowski, $\mathbf{R}$ private communication.

[6] Field, J.B., Toprakcioglu, C., Ball, R.C., Stanley, H.B., Dai, L., Barfield, W., Penfold, J., Smith, G., Hamilton, W. Macromolecules 25, (1992), 434.

[7] Field, J.B., Toprakcioglu, C., Dai, L., Hadziioannou, G., Smith, G., Hamilton, W. J. Phys. II France 2 (1992) 2221.

[8] Auroy, P, Aurvay, L., Léger, L., Phys Rev Let 66, (1991), 719.

[9] de Gennes, P.G. J. Phys. France 36 (1975), 1199.

[10] Grest, G. private communication

[11] Szleifer, I. private communication

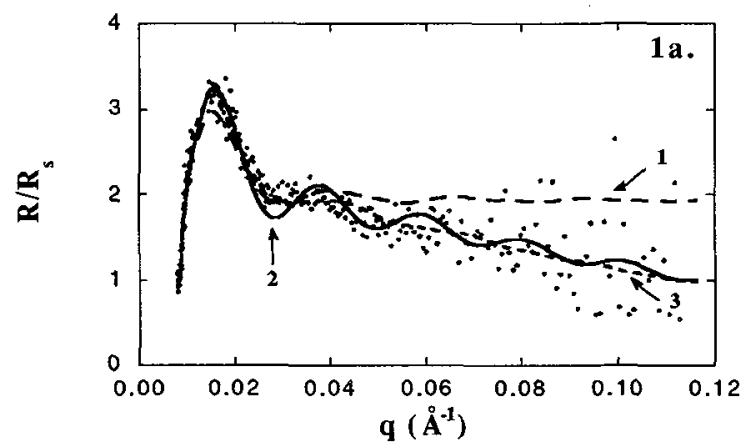

Figure 1. a) Ratio of the reflectivity curve for the monolayer covered surface divided by that for the pure solvent. Solid curves are best fits using the model concentration profiles shown in Figure $1 \mathrm{~b}$. 


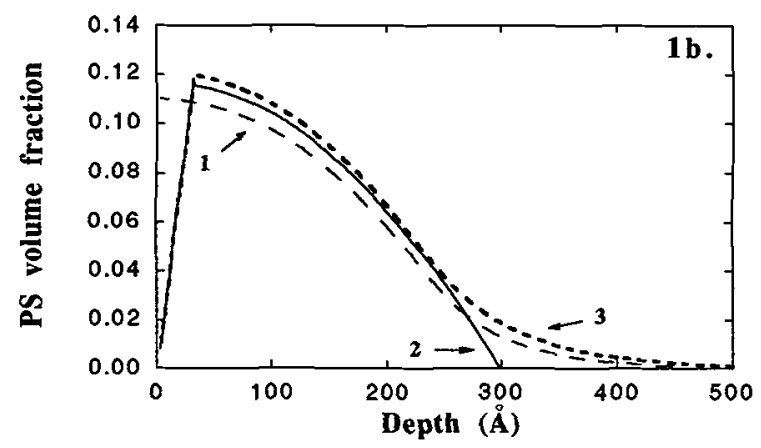

Figure 1b) Best fit profiles corresponding to the curves in Figure 1a. Both a depletion layer and a smooth tail are required to fit the data.

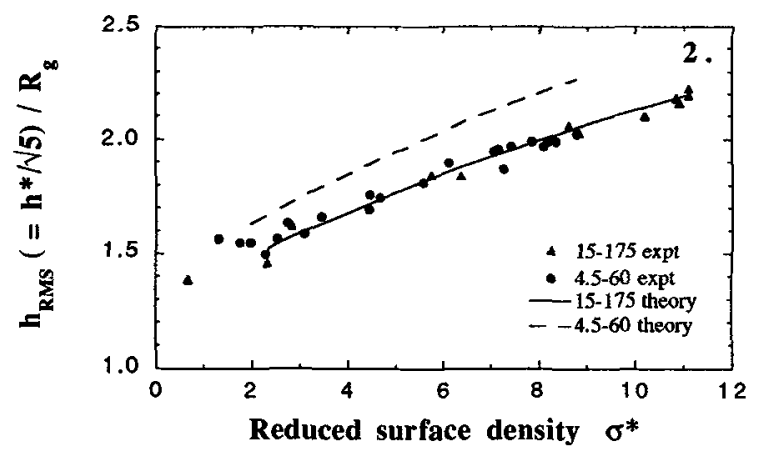

Figure 2. Normalized $h^{*}$ versus reduced surface concentration $\sigma^{*}$ : comparison of experimental data with numerical SCF calculations (5).

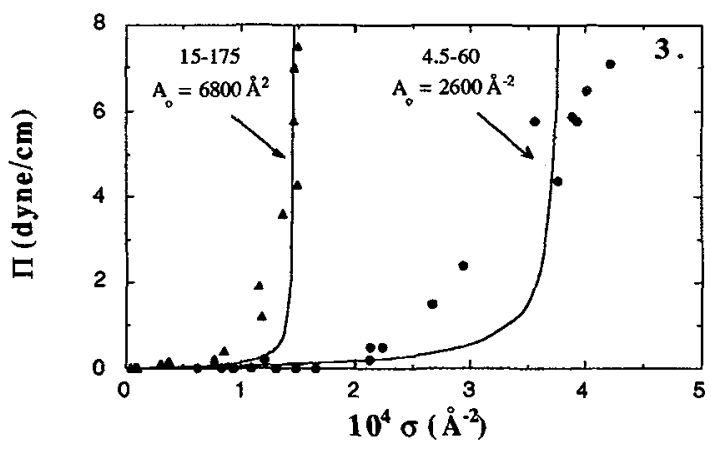

Figure 3. Surface pressure isotherms. Solid lines are calculated using a Van der Waals gas model with hard core interactions. $\mathrm{A}_{0}$ is the hard core cross-sectional area.

Acknowledgments: We would like to thank David Andelman for helpful discussions and Mark Whitmore and Romek Baranowski for performing the SCF calculations. This work was partially supported by the U. S. Department of Energy under contract \#DE-AC04-76DP00789. 